\title{
Critical Dynamics of Contact Line Depinning
}

\author{
Deniz Ertaş and Mehran Kardar \\ Department of Physics \\ Massachusetts Institute of Technology \\ Cambridge, Massachusetts 02139
}

(August 5, 2021)

The depinning of a contact line is studied as a dynamical critical phenomenon by a functional renormalization group technique. In $D=2-\epsilon$ interface dimensions, the roughness exponent is $\zeta=\epsilon / 3$ to all orders in perturbation theory. Thus, $\zeta=1 / 3$ for the contact line, equal to the Imry-Ma estimate of Huse for the equilibrium roughness. The dynamical exponent is $z=1-2 \epsilon / 9+O\left(\epsilon^{2}\right)<1$, resulting in unusual dynamical properties. In particular, a characteristic distortion length of the contact line depinning from a strong defect is predicted to initially increase faster than linearly in time. Some experiments are suggested to probe such dynamics.

Wetting phenomena and contact lines (CLs) appear in many manufacturing processes that involve the spreading of a liquid on a solid surface [1]. Some degree of control over the spreading of the liquid and the corresponding creep of the CL is needed to optimize the desired characteristics of such processes. In particular, it is important to know the effect of surface roughness and contaminants at microscopic to mesoscopic scales on CL dynamics. Here, we study such effects at length scales from $10^{-1}$ down to $10^{-7}$ centimeters. The upper length scale is set by the droplet size or the capillary length (due to gravitation), while the lower length scale is determined by the characteristic size of the microscopic defects.

Surface impurities lead to $C L$ hysteresis, i.e. a finite force is needed to start the fluid spreading. Recently, the scaling exponents of a driven elastic interface subject to quenched impurities near a similar depinning treshold have been calculated through a functional renormalization-group (RG) treatment close to four interface dimensions [2, 3]. Here we apply this method to calculate various scaling exponents for the slowly advancing contact line. The distinction between the two cases is that the CL is the termination of the liquid-vapor interface. We shall assume that the partially wetting fluid spreads sufficiently slowly on a heterogenous surface that the liquid-vapor interface evolves adiabatically, i.e., the it responds to changes in the CL shape instantenously. In this case, fluctuations of the CL around its time-averaged value reflect the competition between impurities on the solid surface and the liquid-vapor surface tension.

Consider a wetting front on a heterogenous surface in the $x-y$ plane with the average orientation of the contact line in the $x$ direction as shown in Fig. . In equilibrium on a homogenous interface, the macroscopic contact angle
$\Theta$ is determined by the Young condition,

$$
\gamma_{S V}-\gamma_{S L}-\gamma \cos \Theta=0
$$

In the above, $\gamma_{S V}, \gamma_{S L}$, and $\gamma$ are the interfacial tensions for the solid-vapor, solid-liquid, and liquid-vapor interfaces, respectively. The heterogeneities (aka defects) on the surface can be modeled as fluctuations in the difference of local interfacial energy densities,

$$
f(x, y)=\gamma_{S V}(x, y)-\gamma_{S L}(x, y)-\left\langle\gamma_{S V}-\gamma_{S L}\right\rangle .
$$

The average of $f$ is zero, while its correlations satisfy,

$$
\left\langle f(x, y) f\left(x^{\prime}, y^{\prime}\right)\right\rangle=\Delta(r / a),
$$

where $r^{2}=\left(x-x^{\prime}\right)^{2}+\left(y-y^{\prime}\right)^{2}, a$ is the characteristic size of defects, and $\Delta$ is a function that decays rapidly for large values of its argument. (For a self-affinely rough surface, $\Delta$ may have a slow algebraic decay [1]. Such situations will not be explored here.) Fluctuations of the CL from its average position, $h_{C L}(t)=v t$, are denoted by $h(x, t)$, and thus satisfy

$$
\overline{\langle h(x, t)\rangle}=0 \text {. }
$$

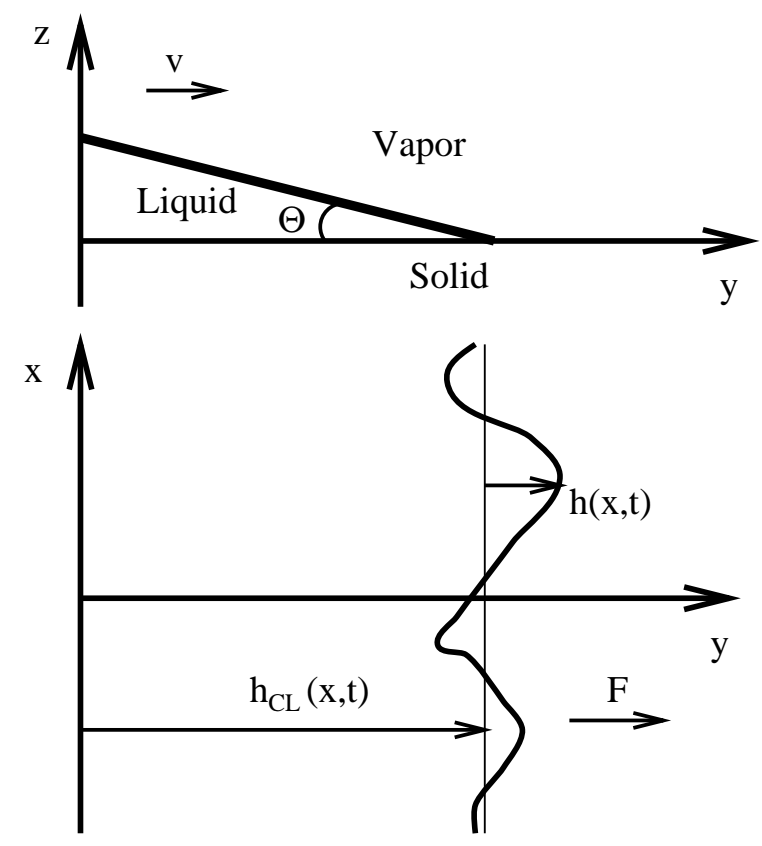

FIG. 1. Geometry of the system. 
(The overline denotes a time average.) The capillary energy associated with small deformations of a CL was calculated by Joanny and de Gennes [4] (in the $\Theta \rightarrow 0$ limit) as

$$
\begin{aligned}
U_{\text {cap }} & =\frac{\gamma \Theta^{2}}{2} \int_{\frac{2 \pi}{a}<|q|<\frac{2 \pi}{L}} \frac{d q}{2 \pi}|q||h(q)|^{2} \\
& =\frac{\gamma \Theta^{2}}{2 \pi} \int_{a<\left|x-x^{\prime}\right|<L} d x d x^{\prime} \frac{h(x) h\left(x^{\prime}\right)}{\left(x-x^{\prime}\right)^{2}},
\end{aligned}
$$

where $h(q)$ is the fourier transform of the contact line profile at a given time, and $a$ and $L$ are the lower and upper cutoff length scales mentioned earlier. The unusual $q$-dependence of the energy functional, and the resulting nonlocal dynamics, reflects the fact that perturbations of wavevector $q$ on the CL induce deformations into the liquid-vapor interface up to a distance $|q|^{-1}$.

A random contribution to the liquid-surface energy comes from the defects [1],

$$
U_{\text {rand }}=\int_{-\infty}^{+\infty} d x \int_{-\infty}^{h_{C L}+h(x)} d y f(x, y) .
$$

If the heterogeneity is strong enough, there is contact angle hysteresis [1. [1. This arises from many metastable configurations of the CL profile, which are given by local minima of the free energy. The surface tensions $\gamma_{S L}, \gamma_{S V}$ in Eq.(11) must then be interpreted as spatial averages over the CL position. Thus, the macroscopic contact angle will depend on the particular CL profile. If a macroscopic force of $F$ per unit length is exerted on the CL, it will move with a finite velocity $v$ only for $F>F_{c}$. This occurs when the metastable state with the largest $\Theta$, usually referred to as the advancing angle $\Theta_{a}$ [1,, , becomes unstable. For small capillary numbers, $\Theta \approx \Theta_{a}$.

As the CL slowly advances, energy is dissipated through various mechanisms [1]. If the dissipation in the vicinity of the CL dominates for small $v$, the drag force at a point $x$ on the CL is simply related to the local velocity $v+\partial_{t} h(x, t)$ through a microscopic mobility $\mu$. In this case, the equation of motion for the CL is obtained by equating the drag force to the applied force, $-\delta U / \delta h(x)$, as

$$
\begin{aligned}
\mu^{-1}\left(v+\frac{\partial h(x, t)}{\partial t}\right)= & -\frac{\gamma \Theta^{2}}{\pi} \int_{a<\left|x-x^{\prime}\right|<L} d x^{\prime} \frac{h\left(x^{\prime}, t\right)}{\left(x-x^{\prime}\right)^{2}} \\
& +f[x, v t+h(x, t)]+F .
\end{aligned}
$$

In the above formula, $v$ is determined self-consistenly by enforcing Eq. (伍). Note that Eq.(17) is only valid for $v>0$, and $F \rightarrow F_{c}$ from above when $v \rightarrow 0$. The dynamical contact angle $\Theta$ is given by the force balance equation,

$$
F-F_{c}=\gamma\left(\cos \Theta_{a}-\cos \Theta\right) .
$$

A recent experiment on depinning from a single defect [5] is in agreement with the deterministic $(f=0)$ form of this dynamics: Upon depinning from the defect, the time evolution of the CL profile is given by

$$
h(x, t) \sim \ln \left(\frac{x^{2}+(c t)^{2}}{L^{2}}\right),
$$

where the width of the logarithmic profile increases linearly with a characteristic velocity $c=\gamma \Theta^{2} \mu$ after depinning.

By analogy to similar previously studied systems [2,3,7], for $F$ slightly above treshold, we expect the average velocity of the contact line to scale as

$$
v=A\left(F-F_{c}\right)^{\beta},
$$

where $\beta$ is the velocity exponent, and $A$ is a nonuniversal constant. Superposed on the steady advance of the CL are rapid "jumps" as portions of the line depin from strong pinning centers. Such jumps are similar to avalanches in other slowly forced systems, and have a power-law distribution in size, cut-off at a characteristic correlation length $\xi$. On approaching the threshold, $\xi$ diverges as

$$
\xi \sim\left(F-F_{c}\right)^{-\nu},
$$

defining a correlation length exponent $\nu$. At length scales smaller than $\xi$, the interface is self-affine, i.e. with correlations satisfying the dynamic scaling form

$$
\left\langle\left(h(x, t)-h\left(x^{\prime}, t^{\prime}\right)\right)^{2}\right\rangle=\left(x-x^{\prime}\right)^{2 \zeta} g\left(\frac{t-t^{\prime}}{\left(x-x^{\prime}\right)^{z}}\right),
$$

where $\zeta$ and $z$ are the roughness and dynamic exponents, respectively. The scaling function $g$ goes to a constant as its argument approaches $0, \zeta$ is the wandering exponent of an instantenous CL profile, and $z$ relates the average lifetime of an avalanche to its size by $\tau(\xi) \sim \xi^{z}$.

In many aspects, Eq.(77) is similar to the model analyzed by Narayan and Fisher using the formalism of Martin, Siggia, and Rose [6] (MSR), through an expansion around a mean field solution [3]. To use this method, it is necessary to generalize to a $D$-dimensional interface for a systematic computation of the exponents through an $\epsilon$ expansion. Introducing an auxiliary field $\hat{h}(x, t)$, the MSR generating functional is,

$$
\begin{gathered}
Z=\int[d h][d \hat{h}] \exp \left\{i \int d^{D} \mathbf{x} d t \hat{h}(\mathbf{x}, t) \mathcal{F}[h, f]\right\}, \\
\mathcal{F}[h, f]=\mu^{-1}\left(\partial_{t} h(\mathbf{x}, t)+v\right)-f[\mathbf{x}, v t+h(\mathbf{x}, t)]-F \\
+\gamma \Theta^{2} \int d^{D} \mathbf{x}^{\prime} K_{D}\left(\mathbf{x}-\mathbf{x}^{\prime}\right) h\left(\mathbf{x}^{\prime}, t\right) .
\end{gathered}
$$

In the above, $K_{D}(\mathbf{x})\left(\sim|\mathbf{x}|^{-(D+1)}\right.$ for $\left.a<|\mathbf{x}|<L\right)$ is the generalized interaction kernel in $D$ dimensions. Mean field (MF) theory is obtained by replacing the capillary forces on a portion of the CL with Hookean springs. This gives 


$$
\mu^{-1}\left(\frac{d h_{M F}}{d t}+v\right)=-k h_{M F}(t)+f\left[v t+h_{M F}(t)\right]+F,
$$

independently for each $\mathbf{x}$, and identical to the mean field model analyzed by NF [3,7]. Here again, $v(F)$ is determined by the self-consistency condition $\overline{\left\langle h_{M F}\right\rangle}=0$.

The MF solution depends on the type of irregularity: For smoothly varying $f(x, y), \beta_{M F}=3 / 2$, whereas for "mesa" defects, i.e. cusped $f(x, y), \beta_{M F}=1$ [7],8]. Some recent experiments on reasonably clean surfaces by Ström et al. [9] are in agreement with Eq.(10) for $\beta=3 / 2$ in the low capillary number regime. Even though it is generally believed that the scaling relation in Eq.(10) holds [10], it is clear that the prefactor is nonuniversal, and various experiments to date have obtained widely varying $v-F$ behaviors 11]. To our knowledge, there are no experiments so far that have systematically investigated strongly heterogenous surfaces with reasonable accuracy.

Following the treatment of NF [3,7], we use the mean field solution for cusped potentials, anticipating jumps with velocity of $O(1)$, in which case $\beta_{M F}=1$. After rescaling and averaging over impurity configurations, we arrive at a generating functional whose low-frequency form is

$$
\begin{aligned}
\bar{Z}= & \int[d H][d \hat{H}] \exp (-S), \\
S= & \int d^{D} \mathbf{x} d t\left[F-F_{M F}(v)\right] \hat{H}(\mathbf{x}, t) \\
& +\int \frac{d \omega}{2 \pi} \frac{d^{D} \mathbf{q}}{(2 \pi)^{D}} \hat{H}(-\mathbf{q},-\omega)(-i \omega+|\mathbf{q}|) H(\mathbf{q}, \omega) \\
& -\frac{1}{2} \int d^{D} \mathbf{x} d t d t^{\prime} \hat{H}(\mathbf{x}, t) \hat{H}\left(\mathbf{x}, t^{\prime}\right) \\
& \times C\left[v t-v t^{\prime}+H(\mathbf{x}, t)-H\left(\mathbf{x}, t^{\prime}\right)\right] .
\end{aligned}
$$

In the above expressions, $H$ and $\hat{H}$ are coarse-grained forms of $h$ and $\hat{h}$, in the sense that response and correlation functions of $h, \hat{h}$ are the same as those of $H, \hat{H}$ at low frequencies. $F$ is adjusted to satisfy the condition $\langle H\rangle=0$. The function $C(v \tau)$ is initially the mean-field correlation function $\left\langle h_{M F}(t) h_{M F}(t+\tau)\right\rangle$.

All non-gaussian terms in the generating functional $\bar{Z}$, including ones that are not shown in Eq.(15), decay away at large length and time scales for $D>D_{c}=2$. Thus, for $D>D_{c}$, exponents can be easily determined from the gaussian theory as $z_{0}=\nu_{0}=\beta_{0}=1$. The interface is flat at long length scales $\left(\zeta_{0}=(2-D) / 2\right)$.

At $D=D_{c}$, there are an infinite number of marginal operators that can be cast into a single marginal function for $v \rightarrow 0$. The RG is carried out by integrating over a momentum shell and all frequencies, followed by a scale transformation $x \rightarrow b x, t \rightarrow b^{z} t, H \rightarrow b^{\zeta} H$, and $\hat{H} \rightarrow$ $b^{\theta-D} \hat{H}$, where $b=e^{l}$. For $D<D_{c}$, there are a number of exact exponent relations [3]:

$$
\beta=(z-\zeta) \nu,
$$

$$
\begin{aligned}
& \nu=1 /(z+\theta), \\
& \nu=1 /(1-\zeta) .
\end{aligned}
$$

The first result comes from the scaling of nonlinear response functions, requiring $H$ to scale as $v t$. The second relation follows from the renormalization of $F-F_{M F}(v)$ through the first term in Eq.15]. Finally, the last relation results from an exact statistical symmetry of the system that fixes the form of the static $(\omega=0)$ response function $\left\langle\partial h\left(x^{\prime}\right) / \partial f(x)\right\rangle$. These relations determine all exponents in terms of $\zeta$ and $z$. The diagrams appearing in the renormalization of $C(u)$ are exactly the same as in Ref. [3], with the exception of the form of the bare propagator, which is $(-i \omega+|q|)^{-1}$ instead of $\left(-i \omega+q^{2}\right)^{-1}$. Thus, the renormalization of $C$ in $D=2-\epsilon$ interface dimensions, computed to one-loop order, gives the recursion relation,

$$
\begin{aligned}
\frac{\partial C(u)}{\partial l}=[ & +2 \theta+2(z-1)] C(u)+\zeta u \frac{d C(u)}{d u} \\
& -\frac{1}{2 \pi} \frac{d}{d u}\left\{[C(u)-C(0)] \frac{d C(u)}{d u}\right\} .
\end{aligned}
$$

$\mathrm{NF}$ showed that all higher order diagrams contribute to the renormalization of $C$ as total derivatives with respect to $u$, thus, integrating Eq.(19) at the fixed-point solution $\partial C^{*} / \partial l=0$, together with Eqs.(17) and (18), gives $\zeta=$ $\epsilon / 3$ to all orders in $\epsilon$, provided that $\int C^{*} \neq 0$. For the case of a CL $(D=1)$, we thus obtain

$$
\zeta=1 / 3, \quad \text { and } \quad \nu=3 / 2 .
$$

This value of the roughness exponent coincides with the Imry-Ma estimate of Huse given in Ref. [1] for the equilibrium roughness. This is a consequence of the fact that $C(u)$ remains short-ranged upon renormalization, implying the absence of anomalous contributions to $\zeta$.

To calculate the dynamical exponent $z$, we need the fixed-point function $C^{*}$, which is obtained only to $O(\epsilon)$ from the above analysis. Furthermore, the calculation requires some knowledge of the high-frequency form of response and correlation functions, i.e., a low-frequency analysis of Eq.(15) is insufficient to describe scaling properties of the system. Nevertheless, NF calculate the exponent $\theta$ to $O(\epsilon)$ as $\theta+\zeta=(\epsilon-\zeta) / 3+O\left(\epsilon^{2}\right)$. The computation for the CL gives the same result, yielding

$$
\begin{aligned}
& z=1-2 \epsilon / 9+O\left(\epsilon^{2}\right), \\
& \beta=1-2 \epsilon / 9+O\left(\epsilon^{2}\right) .
\end{aligned}
$$

Note that $\beta<z$ even though the difference is $O\left(\epsilon^{2}\right)$. Nattermann et al [2] obtain the same results to $O(\epsilon)$ by directly averaging the MSR generating function in Eq.(13). The treatment of NF has the advantage of expanding around a solution with $F_{c} \neq 0$, resulting in a better behaved theory. In particular, systems that are described by vector fields instead of scalar fields, like the treshold critical dynamics of flux lines in three dimensions, require 
the use of their approach, since the expansion has to be made around a solution with $F_{c} \neq 0 \sqrt{12}$.

The above scaling exponents describe a CL advancing at low capillary number on a dirty surface, and also apply to surfaces with microscopic roughness, i.e. with short range slope-slope correlations. The roughness exponent of $\zeta=1 / 3$, equal to its equilibrium value, can be directly measured by microscopic imaging of a slowly advancing CL. A number of macroscopic experimental tests are also possible: the velocity of the CL is given by by $v \sim(F-$ $\left.F_{c}\right)^{\beta}$ with $\beta<1$ (See Fig. ). The root mean square width of the CL profile should increase like $W \sim \xi^{\zeta} \sim v^{-\zeta / \nu \beta}$ as the threshold is approached. These relations can be checked through tensiometric measurements [11, where the capillary force on a solid immersed into a liquid is measured directly.

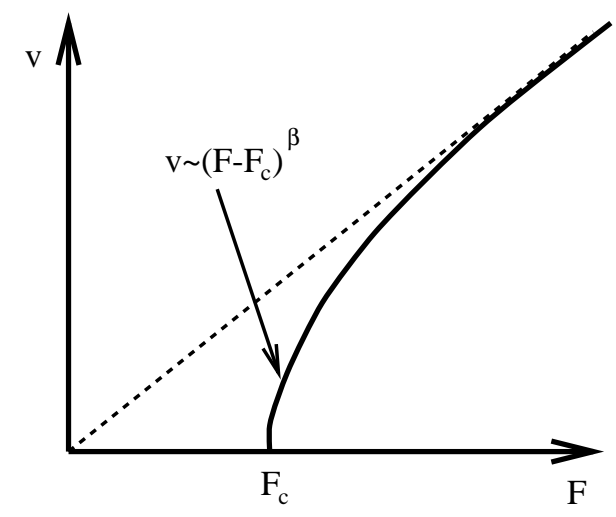

FIG. 2. Predicted velocity-force behavior for a depinning contact line; $\beta=7 / 9$ to first order in $\varepsilon=2-\mathrm{D}$.

A dynamical exponent of $z<1$ suggests that the relaxation of the CL is very different on a dirty surface. Upon depinning from a strong defect, the width of the logarithmic CL profile initially grows faster than linearly in time, in other words, with a characteristic velocity that increases with time: $c(t) \sim t^{(1-z) / z}$. This is, of course, not physical at arbitrarily large length and time scales. We have assumed that the liquid-vapor interface retains its equilibrium shape determined by the CL profile at all times. This picture will break down as characteristic velocities become comparable to capillary wave velocity $c_{\text {cap }}=\sqrt{\gamma / \rho}$ of the liquid-vapor interface. The scaling regime in between should still be accessible to experiments in which the depinning from a single strong defect is observed on a dirty or rough surface.

Finally, NF carried their analysis further to speculate exponents below the treshold when the driving force is increased monotonically towards $F_{c}$ [3]. In particular, they postulated an avalanche distribution

$$
\operatorname{Prob}(\text { width of avalanche }>\ell) \approx \frac{1}{\ell^{\kappa}} \hat{\rho}\left(\ell / \xi_{-}\right),
$$

with $\xi_{-} \sim\left(F_{c}-F\right)^{\nu_{-}}$, and the mean polarizability

$$
\chi_{\uparrow}=\frac{d\left\langle\int h\right\rangle}{d F_{\uparrow}} \sim\left(F_{c}-F\right)^{-\gamma} .
$$

If $\nu_{-}=\nu$, and the scaling hypothesis holds for the CL, it then follows that $\kappa=D-1 / \nu_{-}$, and $\gamma=1+\zeta \nu_{-}$. These exponents are then given exactly by $\gamma=\nu_{-}=3 / 2$, and $\kappa=1 / 3$ in $D=1$.

We thank O. Narayan for various discussions and communicating his results. This research was supported by grants from the NSF (DMR-93-03667 and PYI/DMR-8958061), and the MIT/INTEVEP collaborative program.

[1] P.G. de Gennes, Rev. Mod. Phys. 57, 827 (1985).

[2] T. Nattermann, S. Stepanow, L.-H. Tang, and H. Leschhorn, J. Phys. II France 2, 1483 (1992).

[3] O. Narayan and D. S. Fisher, Phys. Rev. B 48, 7030 (1993).

[4] J.F. Joanny and P.G. de Gennes, J. Chem. Phys. 81, 552 (1984).

[5] J.A. Marsh and A.M. Cazabat, Phys. Rev. Lett. 71, 2433 (1993).

[6] P.C. Martin, E. Siggia, and H. Rose, Phys. Rev. A 8, 423 (1973).

[7] D.S. Fisher, Phys. Rev. B 31, 1396 (1985), O. Narayan and D.S. Fisher, Phys. Rev. B 46, 11520 (1992).

[8] J.F. Joanny and M.O. Robbins, J. Chem. Phys. 92, 3206 (1990).

[9] G. Ström, M. Fredriksson, P. Stenius, and B. Radoev, J. Colloid Interface Sci. 134, 107 (1990).

[10] J.E. Seebergh and J.C. Berg, Chem. Eng. Sci. 47, 4455 (1992). In their notation, $\beta=1 / B$.

[11] R.A. Hayes and J. Ralston, L. Colloid Interface Sci. 159, 429 (1993).

[12] D. Ertaş and M. Kardar (unpublished). 